\title{
Implantation of Left Ventricular Assist Device After Descending Aortic Stent Graft for Mural Thrombus
}

\author{
Aakash Shah, MD,${ }^{1}$ Bella Onwumbiko, BS, ${ }^{1}$ Carlos O. Encarnacion, MD,${ }^{1}$ Eric Krause, MD, ${ }^{1}$ \\ Erik Sorensen, PhD, ${ }^{2}$ Shahab Toursavadkohi, MD, ${ }^{3}$ Bartley P. Griffith, MD, ${ }^{1}$ Zachary N. Kon, MD ${ }^{4}$ \\ David Kaczorowski, MD ${ }^{1}$
}

${ }^{1}$ Division of Cardiac Surgery, University of Maryland School of Medicine, Baltimore, MD, USA; ${ }^{2}$ Department of Clinical Engineering, University of Maryland Medical Center, Baltimore, MD; ${ }^{3}$ Division of Vascular Surgery, University of Maryland School of Medicine, Baltimore, MD, USA; ${ }^{4}$ Department of Cardiothoracic Surgery, New York University Langone Medical Center, New York, NY, USA

\section{ABSTRACT}

In patients with intraluminal thrombus, commonly applied temporary circulatory support modalities are contraindicated secondary to concern regarding distal or proximal (specifically veno-arterial extracorporeal membrane oxygenation) embolization of the thrombus. Therefore, in patients with cardiogenic shock and synchronous intraluminal descending aortic thrombus, support options are quite limited. We report a case of a 66-year-old man in cardiogenic shock, due to an ischemic cardiomyopathy, who also had intramural thrombus with an intraluminal component in the descending thoracic aorta. An endovascular stent graft was inserted inside the aorta over the location of the mural thrombus. This allowed for the placement of an intra-aortic balloon pump (IABP) for pre-operative optimization. After 3 days, a left ventricular assist device (LVAD) was implanted via left anterolateral thoracotomy with hemi-sternotomy, and the IABP was removed. Post-operatively, he had a relatively uncomplicated course without signs of embolic phenomena and ultimately was discharged home. Surveillance computed tomography imaging at 6 months showed no endovascular leak or migration of the stent. This case demonstrates the feasibility of aortic stent graft placement to allow safe insertion of an IABP in the setting of aortic mural thrombus. Furthermore, it demonstrates the safety and feasibility of LVAD implantation after recent aortic stent graft placement.

\section{INTRODUCTION}

Ischemic cardiomyopathy is the most common cause of heart failure in developed nations, which in the acute setting, may present as cardiogenic shock [Briceno 2016]. This potentially fatal complication often is treated both with inotropes

Received fuly 25, 2019; accepted September 10, 2019.

Correspondence: David 7. Kaczorowski, MD, Associate Professor of Surgery, University of Maryland, Division of Cardiac surgery, 110 S. Paca St., 7th Floor, Baltimore, Maryland; 410-328-5842; fax: 410-328-2750 (e-mail: Dkaczorowski@som.umaryland.edu). and vasoactive agents. In some cases, mechanical support is required. Implantable left ventricular assist devices (LVAD) are being used more frequently as either destination therapy or as a bridge to heart transplantation in suitable candidates. However, medical optimization prior to LVAD implantation is paramount to the post-operative success of these patients. The use of intra-aortic balloon pumps (IABP) often is a suitable option for patients who are not optimized with medical management alone [DeVore 2018]. However, IABPs are contraindicated in a number of situations including significant aortic regurgitation, aortic dissection, and the presence of aortic mural or intraluminal thrombi [Krishna 2009]. We report a case in which an aortic stent graft was placed for aortic mural thrombus to allow the safe insertion of an IABP in order to optimize the patient for LVAD insertion. The patient subsequently underwent uneventful LVAD implantation after optimization with the IABP in the setting of the fresh stent graft.

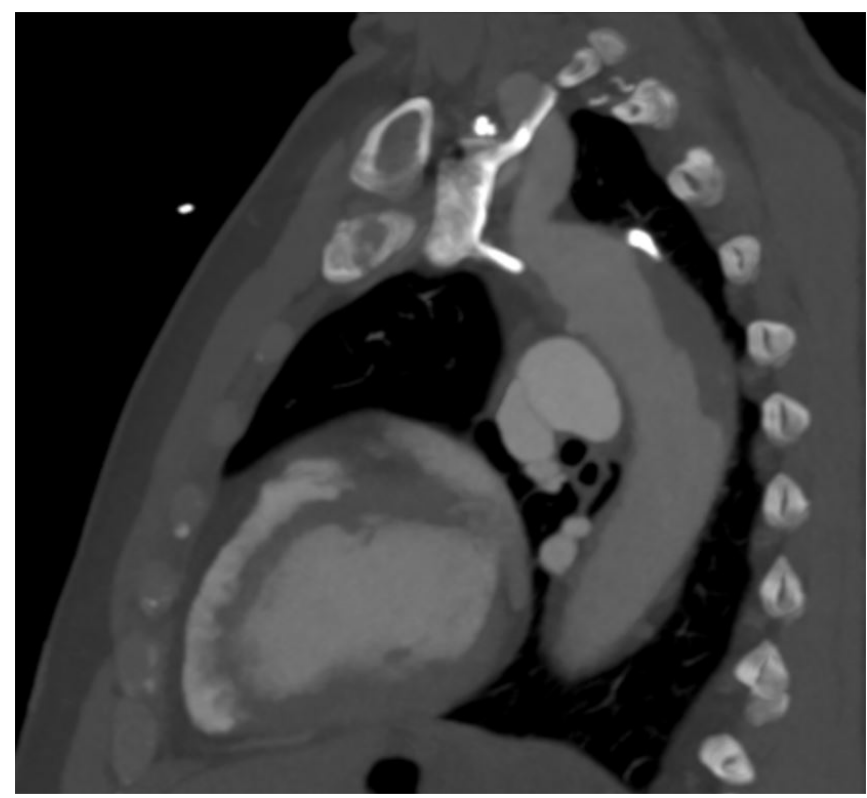

Figure 1. Intramural thrombus with an intraluminal component in the descending thoracic aorta with $40 \%$ luminal narrowing. 


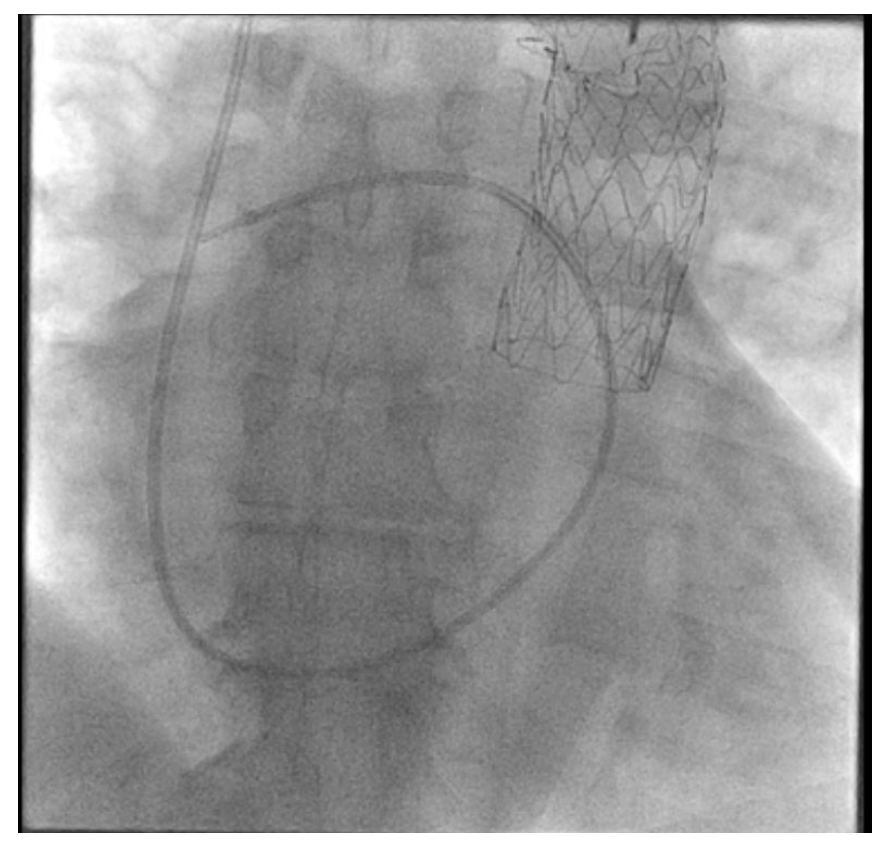

Figure 2. Intra-aortic balloon pump inflated with stent graft in place.

\section{CASE REPORT}

A 66-year-old man with ischemic cardiomyopathy was admitted for cardiogenic shock with malperfusion and multisystem injury, requiring increasing inotropic support. Despite maximal pharmacological therapies, the patient remained insufficiently supported and was deemed to be in need of temporary mechanical support as a bridge to durable left ventricular support. As part of an evaluation for his advanced heart failure and consideration for advanced mechanical circulatory support, he underwent computed tomography imaging, which revealed the presence of an intramural aortic thrombus with an intraluminal component in his proximal descending thoracic aorta (Figure 1). A 31 × 26 × 100 mm Gore-Tag thoracic endovascular stent graft (W. L. Gore and Associates, Flagstaff, Arizona) was deployed just distal to the left subclavian artery via the right common femoral artery under fluoroscopic guidance using standard techniques in order to cover the region of aortic mural thrombus.

Subsequently, a 7.5-Fr IABP was placed via the left common femoral artery. The IABP successfully was inserted without complication (Figure 2). The patient's inotropic requirement began to decrease, and his end-organ dysfunction began to improve, measured by decreasing creatinine, transaminases, and lactate.

Three days later, the IABP was removed and a HeartWare HVAD LVAD (Medtronic, Minneapolis, Minnesota) was inserted via left anterolateral thoracotomy with hemi-sternotomy approach.

Following the procedure, the patient gradually was weaned off of the vasopressor medications and had a relatively uncomplicated course. He was managed with continuous renal replacement therapy for 7 days for his AKI. Ultimately, his urine output improved and his creatinine returned to his baseline of $1.2 \mathrm{mg} / \mathrm{dL}$. He had no signs of embolic phenomena

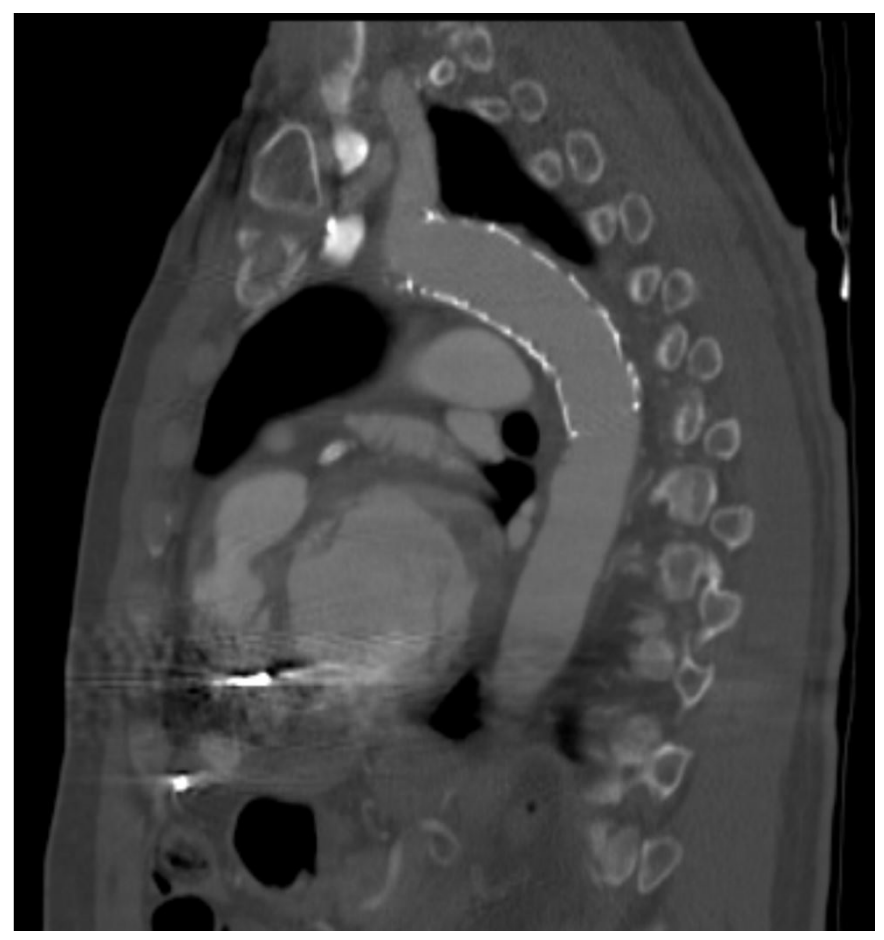

Figure 3. Stent graft in place without endovascular leak 6 months after LVAD implantation.

post IABP and LVAD implantation, such as stroke, mesenteric ischemia, spinal ischemia, or blue-toe phenomenon. He was discharged 19 days post-LVAD implantation.

At his 6 months post-operative follow up, a computed tomography image of the chest showed the stent graft in place, with complete exclusion of the mural thrombus from the circulation and without any signs of migration (Figure 3 ). $\mathrm{He}$ is now more than 14 months out from surgery and has remained free from signs of embolic phenomena.

\section{DISCUSSION}

In a patient suffering from frank cardiogenic shock with worsening end-organ perfusion in spite of inotropic and pressor support, there often is a need for additional temporary mechanical circulatory support as a bridge to durable LVAD. A variety of modalities may be employed, including insertion of IABP, veno-arterial extracorporeal membrane oxygenation (VA-ECMO), or temporary LVAD implantation [Garatti 2007]. However, prior to durable LVAD implantation, these patients must be optimized in order to minimize post-operative complications. The presence of a descending aortic mural thrombus complicates these support options.

Descending aortic mural thrombus has an incidence of $0.45 \%$ in the general population, with $17 \%$ showing evidence of distal embolization and $6 \%$ severe enough to be considered the cause of death [Machleder 1986]. The placement of an IABP is generally thought to be contraindicated as the counterpulsation of the device against the aortic mural 
thrombus may result in distal embolization. Similarly, the use of VA-ECMO in this patient was not a desirable option due to the possibility of retrograde flow potentially leading to stroke. Given these considerations, we felt the mural thrombus should be addressed prior to temporary mechanical circulatory support. Treatment strategies include anticoagulation alone, open thrombectomy, surgical replacement of aorta, and endovascular exclusion. However in these patients, anticoagulation alone may not be reasonable as over $30 \%$ of patients have persistent thrombus [Meyermann 2017]. Also, a patient in cardiogenic shock may not tolerate open surgery, and a less invasive endovascular option is desirable.

We opted for endovascular exclusion of the thrombus and placement of an IABP in the same operative setting. The patient's end-organ perfusion improved over the course of the next 3 days, allowing for safe implantation of LVAD. Importantly, no endoleak or aortic dissection occurred, despite using a partial occlusion clamp on the ascending aorta to fashion the outflow graft anastomosis at the time of LVAD insertion. Lastly, the consequences of implantation of an LVAD with continuous flow in a patient with a recent endovascular stent graft are not known. There is a potential concern for paraplegia given the relative spinal hypoperfusion with the laminar flow from the LVAD in concert with a descending aortic endovascular stent graft. In this case, we observed longterm stability of the stent graft without stent migration, and no signs of neurologic compromise to the lower extremities.

\section{CONCLUSION}

This case represents the successful management of a patient in cardiogenic shock, due to advanced heart failure and synchronous mural thrombus with intraluminal component in the proximal descending aorta. While IABP therapy generally is contraindicated in patients with intraluminal aortic thrombi, IABP placement may be performed with lower risk of embolic complications if an endovascular stent graft is placed over the thrombus prior to IABP insertion. In addition, this case demonstrates the safety of LVAD insertion in a patient with a recent endovascular stent graft, without stent migration or paraplegia.

\section{REFERENCES}

Briceno N, Schuster A, Lumley M, and Perera D. 2016. Ischaemic cardiomyopathy: pathophysiology, assessment, and the role of revascularization. Heart 102(5):397-406.

DeVore AD, Hammill BG, Patel CB, et al. 2018. Intra-aortic balloon pump use before left ventricular assist device implantation: Insights from the INTERMACS registry. ASAIO J 64(2):218-224.

Garatti A, Russo C, Lanfranconi M, et al. 2007. Mechanical circulatory support for cardiogenic shock complicating acute myocardial infarction: an experimental and clinical review. ASAIO J 53(3):278-87.

Krishna M, and Zacharowski K. 2009. Principles of intra-aortic balloon pump counterpulsation. Continuing Education in Anaesthesia Critical Care \& Pain 9(1):24-28.

Machleder HI, Takiff H, Lois JF, Holburt E. 1986. Aortic mural thrombus: an occult source of arterial thromboembolism. J Vasc Surg 4:473-78.

Meyermann K, Trani J, Caputo FJ, Lobardi JV. 2017. Descending thoracic aortic mural thrombus presentation and treatment strategies. J Vasc Surg 66(3):931-36. 